\title{
Value of Minimally Invasive Gynecologic Surgeon in Surgical Management of Endometriosis
}

\author{
Natasha Gupta, MD* \\ Department of Obstetrics and Gynecology, Minimally Invasive Gynecologic Surgery, Jackson Madison \\ County General Hospital, Jackson, USA
}

*Corresponding author: Natasha Gupta, MD, Department of Obstetrics and Gynecology, Minimally Invasive Gynecologic Surgery, Jackson Madison County General Hospital/Woman's Clinic P.A, 244 Coats land Drive, Jackson, TN, 38301, USA, Tel: +1-248-464-0451

\begin{abstract}
The quality of endometriosis surgery performed by a Minimally Invasive Gynecologic Surgeon (MIGS) is significantly different from that of a general gynecologist. Their training offers them the experience to detect subtle endometriosis, excise deeply infiltrative endometriosis, manage extra pelvic endometriosis and restore normal anatomy in stage 4 endometriosis. They are able to preserve fertility in an endometriosis patient if patient desires fertility. They are also able to address or excise endometriosis when performing concurrent hysterectomy, instead of disregarding it.

In this article, a unique perspective of a fellowship trained MIGS is presented who practices as the only MIGS for that area. As a result, there is no dearth of inadequately managed endometriosis patients. This article highlights a unique scenario where inability to excise ovary completely during an oophorectomy in a stage 4 endometriosis patient will lead to ovarian remnant syndrome.

The paucity of generalist's experience in surgical management of endometriosis leads to unnecessary hysterectomies, inability to recognize endometriosis in early stages, inappropriate treatments like fulguration and rare conditions like ovarian remnant.
\end{abstract}

\section{Introduction}

I trained in a Minimally Invasive Gynecologic Surgery (MIGS) fellowship program where we were referred the patients with chronic pelvic pain and endometriosis. Needless to say, these are the patients that have been given the 'run around' for management of pelvic pain. The typical treatment course of these patients consists of diagnostic laparoscopy; if fortunate, they may receive fulguration of endometriotic implants followed by GnRH agonists or some form of birth control [1]. Predictably, patient has recurrent pain and gets a few more laparoscopies. If lucky, they finally escape their general gynecologic surgeon and finally seek help from a specialist in Minimally Invasive Gynecologic Surgery (MIGS). If not that lucky, they end up with a hysterectomy without any concurrent excision of endometriosis. Reproductive age females desirous of fertility end up choosing hysterectomy due to persistent pelvic pain, dysfunctional lifestyle and psycho-social problems caused by the chronic non-menstrual pelvic pain. Many of these patients have subtle, non-text book appearance of endometriosis which gets overlooked by the general gynecologists who only acknowledge the typical black appearance of endometriotic implants.

As a fellowship trained MIGS who joined a general obstetrics and gynecology practice, I have seen variety of ways in which endometriosis patients are 'managed' [2]. Most of the times these patients present with persistent pain despite hysterectomy and bilateral salpingo-oophorectomy. One of the aftermaths of inadequate surgical management is ovarian remnant [3].

I have had a flood of referrals for ovarian remnant excisions. The typical scenarios consist of extensive deeply infiltrative endometriosis in the primary surgery where general gynecologic surgeon naturally unable to dissect the pelvic side walls, endometriomas etc., plucks the uterus and part of ovaries out. Such patients have persistent pelvic pain due to inadequate excision of endometriosis as well as from ovarian remnant syn-

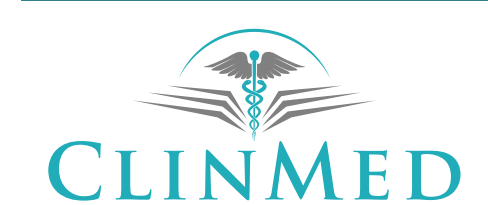

INTERNATIONAL LIBRARY

Citation: Gupta N (2020) Value of Minimally Invasive Gynecologic Surgeon in Surgical Management of Endometriosis. Obstet Gynecol Cases Rev 7:175. doi.org/10.23937/2377-9004/1410175

Accepted: September 29, 2020: Published: October 01, 2020

Copyright: (c) 2020 Gupta N, et al. This is an open-access article distributed under the terms of the Creative Commons Attribution License, which permits unrestricted use, distribution, and reproduction in any medium, provided the original author and source are credited. 
drome [4]. Ovarian remnant is diagnosed typically by MRI or ultrasound which shows persistent ovarian tissue on one or both sides. While performing the ovarian remnant excision in such patients, I come across extensive endometriosis, adhesions, distorted anatomy and retroperitoneal fibrosis.

The excision of endometriosis, restoration of normal anatomy, pelvic side wall dissection, ureterolysis, retroperitoneal dissection, uterine artery ligation at its origin, dissection of rectovaginal space etc., are all the great tools that a MIGS surgeon toils to learn in their fellowship. However, these are elusive to general gynecologist and hence follow this suboptimal management of deeply infiltrative endometriosis.

The lack of proper recognition of MIGS as a board certified subspeciality perpetuates this behavior of general gynecologist. As a MIGS practicing in a small town where I am the only fellowship trained MIGS surgeon, I get referrals for extremely complicated pelvic surgeries. But that's all the referrals I get- only the very challenging cases... There are no slightly or moderately complex cases! Because they are managed by the generalists... somehow... I see cases for fulguration of endometriosis or hysterectomy for endometriosis being performed around me but never a single intraoperative consult for management of the same. That is where I could make a difference with my skills but those are lost to me. And yet I see countless consults for ovarian remnants after a hysterectomy for endometriosis or management of chronic pelvic pain after 4-5 laparoscopies for endometriosis. I believe this is due to lack of awareness as to the tricks and tools that MIGS surgeon can employ and lack of any standard of care in surgical management of deeply infiltrative endometriosis.
In a nutshell, I see three stages of endometriosis in patients-

'Subtle' that a generalist will not be able to identify,

'Black implants' that they will fulgurate and,

'Stage 4 endo' that they will do hysterectomy somehow.

None of those treatment options are optimal...

I believe we can help the women suffering due to the ignorance and inability of their surgeons in managing this particular condition by presenting more updated data of the MIGS performed endometriosis excision, increased incidence of ovarian remnant due to suboptimal excision of endometriosis and set standard of care for such surgeries to be performed by a MIGS trained gynecologic surgeon.

\section{Conflict of Interest}

None.

\section{References}

1. Roman JD (2010) Surgical treatment of endometriosis in private practice: Cohort study with mean follow-up of 3 years. J Minim Invasive Gynecol 17: 42-46.

2. Chopin N, Vieira M, Borghese B, Foulot $H$, Dousset $B$, et al. (2005) Operative management of deeply infiltrating endometriosis: Results on pelvic pain symptoms according to a surgical classification. J Minim Invasive Gynecol 12: 106-112.

3. Wasson MN, Magrina J (2019) Surgical management and prevention of ovarian remnant. J Minim Invasive Gynecol 26: 811 .

4. Kho RM, Abrao MS (2012) Ovarian remnant syndrome: Etiology, diagnosis, treatment and impact of endometriosis. Curr Opin Obstet Gynecol 24: 210-214. 